Article

\title{
Improved Atomization via a Mechanical Atomizer with Optimal Geometric Parameters and an Air-Assisted Component
}

\author{
Inna Levitsky ${ }^{1, *}$ and Dorith Tavor ${ }^{1,2, *}$ \\ 1 Department of Chemical Engineering, Shamoon College of Engineering, PO Box 950, Beer-Sheva 84100, Israel \\ 2 Green Processes Center, Shamoon College of Engineering, PO Box 950, Beer-Sheva 84100, Israel \\ * Correspondence: levii2@sce.ac.il (I.L.); dtavor@sce.ac.il (D.T.)
}

Received: 11 May 2020; Accepted: 8 June 2020; Published: 11 June 2020

\begin{abstract}
Atomization of liquid media is a key aim in various technological disciplines, and solutions that improve spray performance, while decreasing energy consumption, are in great demand. That concept is very important in the development of liquid fuel spray atomizers in high-efficiency microturbines and other generator systems with low inlet pressure and a wide range of power supply. Here we present a study of the liquid atomization characteristics for a new mechanical atomizer that has optimal geometric parameters and a preliminary swirl stage. In our air-assisted atomizer, air is introduced through a swirl chamber positioned at the exit of the mechanical atomizer. The optimized mechanical atomizer alone can achieve $D_{32}$ drop diameters in the range of 80 to $40 \mu \mathrm{m}$ at water supply pressures of 2 to 5 bar, respectively. The addition of an air swirl chamber substantially decreases drop sizes. At an air-liquid ratio (ALR) equal to 1, water pressures of 2.5 to 3 bar and air supply pressures 0.35 to 1 bar, $D_{32}$ drops with diameters of 20-30 $\mu \mathrm{m}$ were obtained. In an air-assisted atomizer the parameters of the mechanical atomizer have a much stronger influence on drop diameters than do characteristics of the air-swirl chamber. Using a mechanical atomizer with optimal geometrical dimensions allows limiting the liquid supply pressure to 5 bar; but when an air-assisted component is introduced we can recommend an ALR $\approx 1$ and an air supply pressure of up to 1 bar.
\end{abstract}

Keywords: mechanical atomizer; air-assisted atomizer; swirl chamber; drop diameters; air/liquid mass flow ratio

\section{Introduction}

Atomization of liquid media is an essential process in many different industries, so solving the technological challenges it presents is of significance for a wide range of applications. Efforts to improve the atomization process are being made in order to increase combustion efficiency in internal combustion gasoline and diesel engines [1,2], to reduce the cost of air humidification and cooling in hothouses [3-5], to aid in air purification [6,7], to improve firefighting appliances while minimizing water usage [8], and to achieve better product solidification for food industries [9]; liquid atomization is also used in the production of pharmaceuticals [10], in the treatments of plants and field for agriculture [11], in textile and tobacco-processing plants [12], and many others.

The atomization of the liquid is accomplished via various atomizer designs. The current designs for liquid atomization may be broadly classed into the following groups:

- Centrifugal nozzles, in which the working medium is given a tangential velocity component and exits through an outlet coaxial with the swirl chamber [13,14].

- Nozzles with impediments, in which the working medium discharges from the nozzle under high pressure and interacts with an obstacle installed in front of the nozzle $[15,16]$. Both of the above 
atomizer types use only the working pressure of the medium transformed into flow velocity to break the liquid up into small-sized particles. Such atomizers are described as mechanical.

- Atomizers that use gaseous media (air, steam, gas) to atomize the liquid. Such atomizers are of the air-assisted (two-phase) type [17-19].

The main benefit of pressure-swirl atomizers is their high energy-efficiency, and many industrial processes use them because of their combination of reliability, ability to achieve droplets of small dimensions, and high performance [20]. In many widely used pressure swirl atomizers, a liquid enters the swirl chamber through a number of tangential holes or slots. Centrifugal force causes the liquid to spread within the chamber as a hollow conical spray, with spray angles ranging from $30^{\circ}$ to almost $180^{\circ}$ depending on the application. Atomization occurs not only because of the break-up of the liquid sheet but also because of collisions between droplets and the interaction between droplets and air [21-23]. The finest atomization occurs at high pressures and at wide spray angles [24]. Atomizer performance has been found to be related to physical and experimental properties such as surface tension, viscosity, mass flow rate, density and injection pressure [25-28]. The mean diameter of drops is strongly associated with the injection pressure. With an increase in the pressure-drop across the atomizer, the liquid exits from the nozzle with a greater velocity, which then creates more intense disturbances on the liquid surface, increasing the quality of atomization. The effect of varying the injection pressure is usually more visible at low injection pressures than at high injection pressures [29]. Rashad et al. found that increasing the injection pressure from 8 bar to 12 bar decreases the $D_{32}$ drop diameters from $69 \mu \mathrm{m}$ to $55 \mu \mathrm{m}$ [30]. However, if increasing pressure from 2 to 10 bar reduces the drop diameters by $45 \%$, then an increase from 10 to 20 bar reduces the drop diameters by $28 \%$ and from 20 to 90 bar the reduction rate is just $42 \%$ [31]. It has been found that there exists a critical injection pressure of 15 bar beyond which the spray angle and drop sizes become practically independent of the pressure [32].

The geometrical parameters of the atomizer, such as the exit diameter of the swirl chamber $\left(d_{n}\right)$ and its length $\left(l_{0}\right)$, the swirl chamber diameter $\left(D_{S}\right)$ and its length $\left(L_{S}\right)$, the atomizer characteristic $(A)$ and inlet tangential cross sectional area $\left(F_{t}\right)$, have direct effects on the swirling motion inside the swirl chamber and consequently are characteristics of the atomization process [33,34]. Chen et al. studied the influences on drop sizes of the ratio of length to swirl chamber diameter $\left(L_{S} / D_{S}\right)$ the number of feed slots, the injection pressure, and the liquid's viscosity, and concluded that an increase in the $L_{S} / D_{S}$ ratio and liquid viscosity decreases the swirling motion [35]. The relation $L_{S} / D_{S}$ should be low to minimize any friction loss. However, the swirl chamber should be designed with a proper height to separate the streams flowing out through the liquid inlet ports. For many designs, an $L_{S} / D_{s}$ ratio ranging from 0.5 to 1.0 is assumed, although it was suggested that higher values of $L_{s} / D_{s}$, up to 2.75 , might improve atomization [36]. Observations have indicated that the $D_{32}$ decreases discernibly and continuously with decreasing $l_{0} / d_{n}$, with the effect on $D_{32}$ in the range of $0.4-2.82 \mu \mathrm{m}$ [37]. The drop diameters also diminished with a reduction of the inlet port area $\left(F_{t}\right)$ [38]. The length of the tangential channels $\left(l_{t}\right)$ of the swirl chamber also affects the characteristics of the atomizer. Short inlet channels are not effective and, if the channel is not long enough, the flow cannot be tangential, and will instead deflect to the axis of the swirl chamber. This deflection will decrease the momentum, increase the discharge coefficient, decrease the spray angle and diminish the quality of atomization. It may be concluded that beginning from $l_{t} / d_{t}>2$, the value of the discharge coefficient and spray angle remains constant, where $d_{t}$ is the diameter of tangential channels [39].

Another geometrical parameter is the convergence angle $\alpha$ of the swirl chamber. Considerable research findings indicate that the convergence angle has an inverse effect on performance parameters, with film thickness and discharge coefficient increasing with the increase of angle $\alpha$. Increasing the angle of the swirl chamber to the nozzle from $60^{\circ}$ to $90^{\circ}$ increases the nozzle discharge coefficient from 0.3 to 0.35 . That said, in cases when the spray angle is smallest and the film thickness is largest at a convergence angle of $90^{\circ}$, this geometry might be preferred as it is easier and less expensive to manufacture compared to a geometry with a smaller convergence angle [40]. 
The atomization of liquid by a mechanical atomizer can be improved by introducing a swirl-air component. Air-assisted atomizers have many advantages due to their ability to work at relatively low fuel supply pressures and to produce finer spraying. Rizkalla and Lefebvre reported that $D_{32}$ becomes smaller with the increase of air velocity, air/liquid ratio, and air density [41]. Fraser et al. found that the air-to-liquid mass flow ratio has little effect on $D_{32}$ for ratios exceeding 1.5 [42]. Those investigations describe a two-phase atomizer, in which after the pressurized liquid exits from the chamber the air is delivered as spray through the radial channels. When the vane angle is $45^{\circ}$, it was shown that the air-pressure drop has little effect on droplet size, if the drop in pressure exceeds 0.02 bar. However, the droplet diameter decreases drastically when the air pressure drop grows from 0 to 0.02 bar [43]. An analysis of the operational parameters of two-phase atomizers shows that the air supply pressure is 2 bar and above. For example, the atomizer of the JS company operates at 2.2 bar, that of the PNR company at a range of 2-4 bar, that of the Lechler company at 2 bar, that of Armstrong at 9.3 bar, and that of Optiquide Ltd between 6 and 8 bar. It should be noted that the compressed air supply pressure has the main effect on the energy consumption of the two-phase atomizers. Development of an atomizer for liquid fuel spray that will operate in a wide range of flow rates and low inlet pressure will reduce the energy consumption in microturbines and other generators.

The current work is a study of our mechanical atomizer with optimized geometric parameters, conducted as the first stage of the research, followed by an investigation of the utility of adding to the system an air supply through a swirl chamber having tangential channels of various diameters. The effect of the interaction of the liquid spray with the swirling air is investigated while changing the various operational and geometrical characteristics of the atomizer.

\section{Materials and Methods}

\subsection{Mechanical Atomizer}

The mechanical atomizer design is presented in Figure 1a. The atomizer is comprised of a swirl chamber with tangential ducts made in separate bush $\{2\}$. The bush is installed in the body $\{1\}$ with the output nozzle of the chamber sealed from the inlet cavity by an o-ring $\{4\}$ and compressed by the bush $\{2\}$. The water is supplied to the atomizer through a nosepiece $\{3\}$.

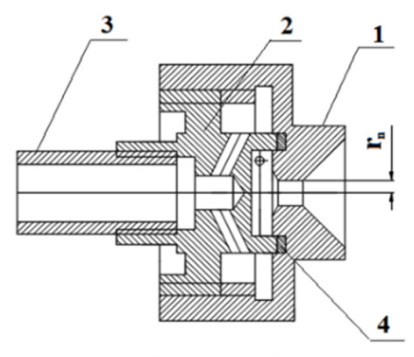

(a)

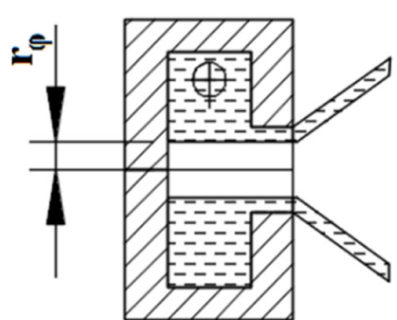

(b)

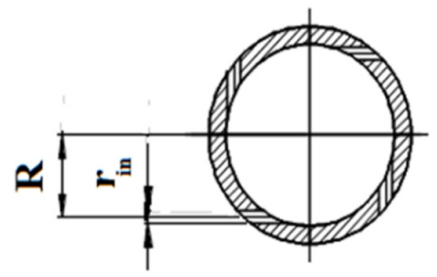

(c)

Figure 1. Mechanical atomizer (a) Atomizer assembly: 1-body, 2-swirl chamber, 3-adapter, 4-o-ring; (b) flow structure; (c) swirl chamber geometry. 
The characteristics of the liquid sheet emanating from the atomizer depend on the geometrical parameters of its swirl chamber. These can be expressed in dimensionless form as atomizer characteristic $A$, and defined as [39]:

$$
A=\frac{R \cdot r_{n}}{n \cdot r_{i n}^{2}}
$$

where $r_{n}$ is the radius of the discharge nozzle (Figure $\left.1 \mathrm{a}\right), R$ is the swirl radius, $r_{i n}$ is the radius of the tangential inlets (Figure 1c), and $n$ is their number. The characteristic $A$ is usually defined as the ratio of the axial flux of angular momentum to the axial linear momentum flux, which in this case is closely approximated by $A=U_{t} / U_{a x}$, where $U_{t}$ and $U_{a x}$ are the liquid tangential and axial velocities at the nozzle exit. According to the theory of the centrifugal nozzle, the impact of characteristic $A$ on the velocities' ratio is considerable up to the values of $A=2.5-4$, after which the rise in $A$ has little effect on the liquid's flow rate coefficient and spray angle [39]. Increasing it further leads to rising hydrodynamic losses in the swirl chamber due to the friction of the flow against the end surfaces, which also lowers the ratio between the tangential and axial velocities, increases the flow rate coefficient, and diminishes the spray angle. Hence, for an available value of inlet pressure, the geometry of the above-listed parameters must be set so as to provide for the highest value of tangential velocity, while reducing the diverse hydrodynamic losses. In view of the above, the geometrical parameters of the experimental atomizer were optimized at the following values: $R=2 \mathrm{~mm}, L_{s}=1.5 \mathrm{~mm}, r_{n}=0.4 \mathrm{~mm}, r_{i n}=0.25 \mathrm{~mm}$, $n=4, A=3.2$. This geometry was adopted in order to provide a water flow rate of $m_{w} \approx 2.0 \times 10^{-3}-2.5$ $\times 10^{-3} \mathrm{~kg} / \mathrm{s}$ with a supply pressure of 2 bar. In the atomizer we developed, $L_{s} / D_{s}$ was equal to $0.3, l_{0} / d_{n}$ $=2, l_{t} / d_{t}=2$, and the convergence angle $\alpha$ of the swirl chamber was equal to $90^{\circ}$.

\subsection{Air-Assisted Atomizer}

The design of the air-assisted atomizer is shown in Figure 2. The atomizer consists of a mechanical atomizer with a swirl chamber $\{1\}$ and bush $\{7\}$, and with tangential channels for introducing air at the exit of the mechanical atomizer. Water enters the swirl chamber from collector $C$ through the tip $\{9\}$ and the radial channels $\{10\}$.
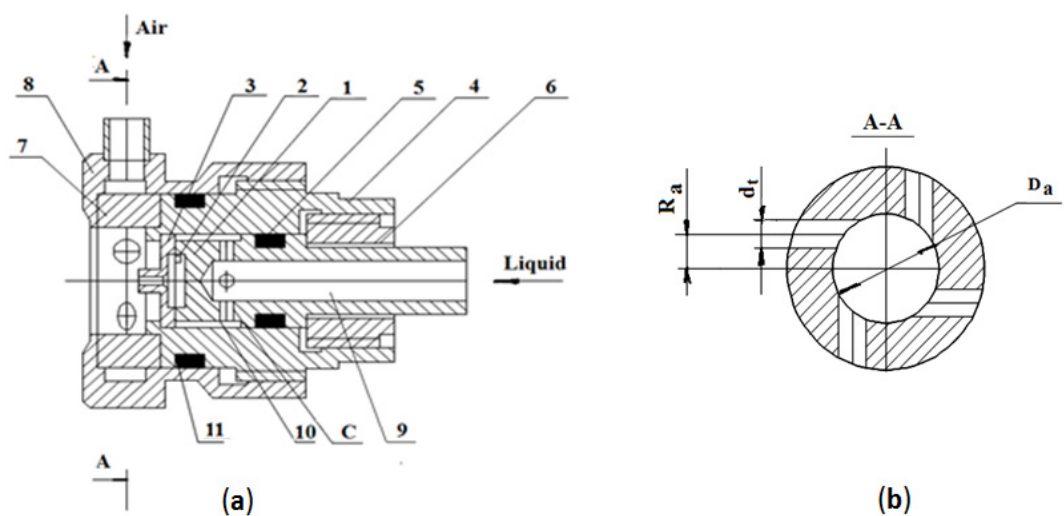

(b)

Figure 2. Design of an air-assisted atomizer. (a) Atomizer assembly: 1-swirl chamber, 2-grooves, 3-nozzle, 4-body, 5, 11-sealing ring, 6-nut, 7-bush, 8-adapter, 9-tip, 10-radial channels.

(b) Cross section of the air swirl chamber.

This design, with the output channel of the mechanical atomizer positioned as a separate part $\{3\}$, is meant to create the possibility of changing the diameter of the swirl chamber outlet nozzle so as to study the effect of various channel diameters on spray quality. The swirl chamber has a nozzle $\{3\}$ pressed to the edge of the body $\{4\}$ by nut $\{6\}$, with the cavity sealed with a ring $\{5\}$. Body $\{4\}$ holds in place the swirl chamber $\{7\}$ installed in the adapter $\{8\}$. The swirl chamber $\{7\}$ is of an open design and contains four tangential channels (Figure $2 b$ ). The region for installing the swirl-air chamber $\{7\}$ is sealed with a ring $\{11\}$. 
The tangential ducts of the swirl chamber in the mechanical atomizer take the form of two grooves, of width $b$ and the depth $h$ (Figure $3 a, b$ ); the water is subsequently let out via the channel with diameter $d_{n}$ (Figure $3 \mathrm{c}$ ) in the nozzle (Figure $2 \mathrm{a}-3$ ).

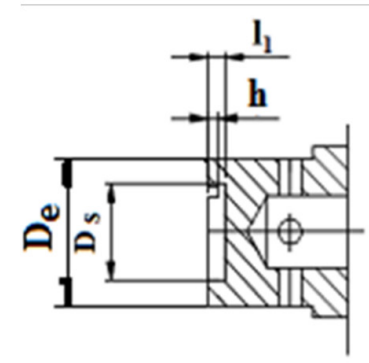

(a)

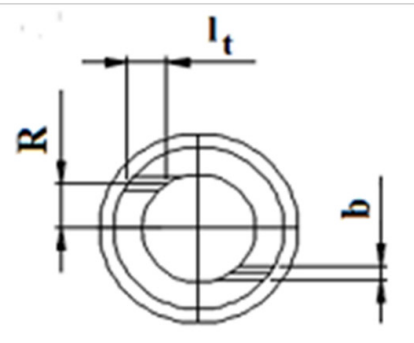

(b)

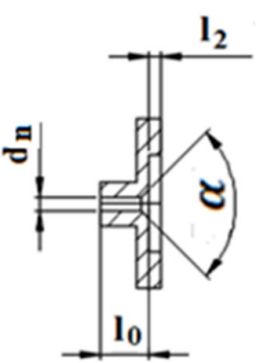

(c)

Figure 3. The geometrical parameters of a pressure swirl atomizer: (a) swirl chamber of a mechanical atomizer, (b) front view of the swirl chamber, (c) nozzle of the swirl chamber.

To obtain an optimal geometry for the mechanical atomizer, the diameter of the swirl chamber $D_{s}$ was set to be $4 \mathrm{~mm}$, the width of the grooves (equal to 2) $b=0.5 \mathrm{~mm}$, depth $h=0.4 \mathrm{~mm}$, the swirl radius $R=1.65 \mathrm{~mm}$, and the outer diameter $D_{e}=6 \mathrm{~mm}$ (Figure 3). The height of the swirl chamber was set at $L_{s}=l_{1}+l_{2}=2 \mathrm{~mm}$. The nozzle exit channel was set to have diameters $d_{n}=0.6$ and $0.8 \mathrm{~mm}$, and length $l_{0}=2 \mathrm{~mm}$. At these dimensions, the parameters were $L_{s} / D_{s}=0.5 ; l_{0} / d_{n}=3.3\left(d_{n}=0.6 \mathrm{~mm}\right)$ and $2.5\left(d_{n}=0.8 \mathrm{~mm}\right) ; l_{t} / b \approx 2$. The convergence angle of the swirl chamber was $\alpha=90^{\circ}$. In line with the derivation of geometrical dimensions of Equation (1), it was determined that $A=3.88$, with a nozzle exit channel $d_{n}=0.6 \mathrm{~mm}$, and $A=5.18$ with $d_{n}=0.8 \mathrm{~mm}$. The air swirl chamber was adjusted to have diameter $D_{a}=7 \mathrm{~mm}$ and tangential channels $(n=4) d_{t}=1.8,2$ and $2.4 \mathrm{~mm}$ and $R_{a}=2.2 \mathrm{~mm}$ (Figure 2b). The geometrical characteristics $A_{a}$ of an air swirl chamber are as follows: $A_{a}=2.4$ at $d_{t}=1.8 \mathrm{~mm} ; A_{a}=1.9$ at $d_{t}=2 \mathrm{~mm}$; and $A_{a}=1.34$ at $d_{t}=2.4 \mathrm{~mm}$.

\subsection{Experimental Setup and Methodology}

Atomization parameters were measured by the TSI application software (TSI Inc., Co, Shoreview, MN, USA), which is part of the TSI phase Doppler particle analyzer/laser Doppler velocimeter (PDPA/LDV) system. PDPA is an optical technique based on an LDV, which allows simultaneous measurement of sizes and velocities of spherical particles by use of a coherent laser beam. The basics of the TSI PDPA/LDV measurement system are described elsewhere [44,45].

The experimental set-up is presented in Figure 4. Measurements of spray characteristics were carried out $50 \mathrm{~mm}$ downstream from the atomizer tip with $5 \mathrm{~mm}$ steps perpendicular to the spray cone diameters. According to Valencia-Bejarano et al., a distance of $50 \mathrm{~mm}$ is sufficient to form droplets but not sufficient to affect droplet size by coalescence [46]. The system automatically measured the drop diameters, after entering the measurement volume, which is less than $10^{-3} \mathrm{~mm}^{3}$. The spray angle was measured based on the liquid flux distribution and digital photographs.

The atomizer's water and air flow rates were obtained in the tests, as well as histograms for the drop diameter distribution under inlet water pressure values of $P_{w}=2-7$ bar in the experimental mechanical atomizer. During the experiment with the two-phase atomizer, the presented characteristics were obtained with a water pressure of 2-3.5 bar and air supply pressures of $0.35,0.7$ and 1 bar.

The resulting data were used for calculation of the mean $D_{32}$ drop diameter, defined by the following relation:

$$
D_{32}=\frac{\sum_{i=1}^{N} D_{i}^{3}}{\sum_{i=1}^{N} D_{i}^{2}}
$$


Here, $D_{32}$ is the Sauter mean diameter, which gives the mean diameter value in terms of the volume/surface ratio.

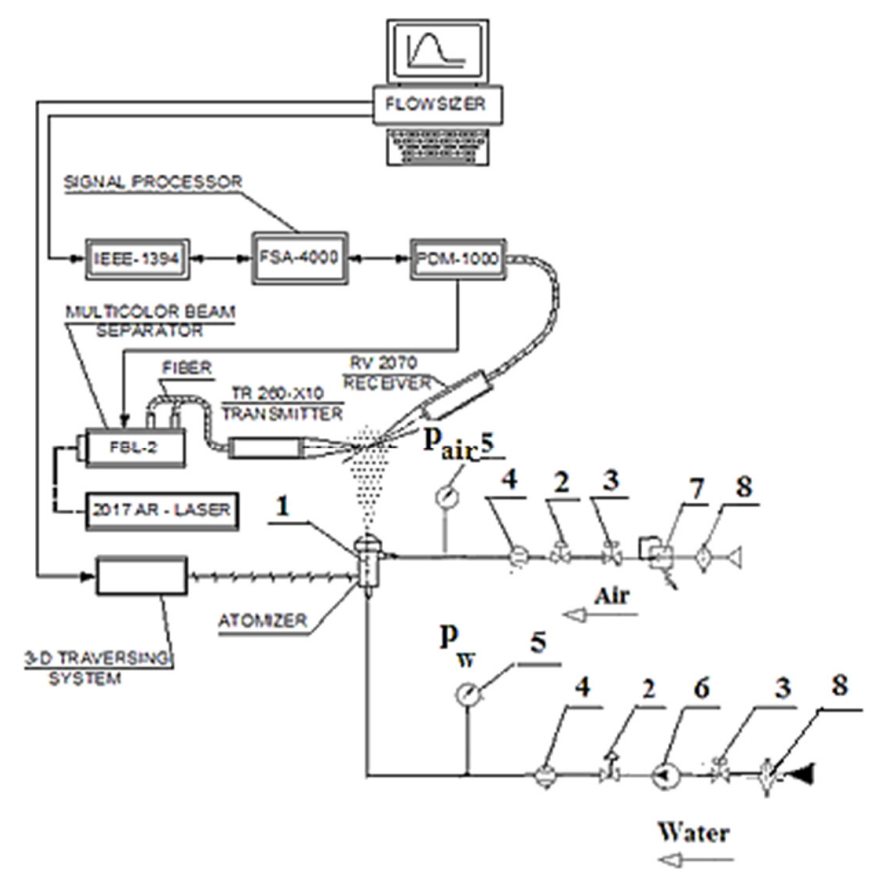

Figure 4. A scheme of the test rig: 1-atomizer, 2-control valve, 3-shut-off valve, 4-flow meter, 5-pressure gauge, 6-water pump, 7-air pressure regulator, 8-filter.

\section{Results and Discussion}

\subsection{The Mechanical Atomizer}

The mechanical atomizer was designed to have optimal geometrical parameters, taking into account data from numerous publications regarding the minimal value of the flow coefficient and thus providing a high-quality liquid spray. Using the test results the flow rate coefficient $\left(C_{d}\right)$ was calculated by Equation (3) [39]:

$$
C_{d}=\frac{Q_{w}}{\pi r_{n}^{2} \sqrt{\frac{2}{\rho_{w}} P_{w}}}
$$

where $Q_{w}$ is water volume flow rate $\left(\mathrm{m}^{3} / \mathrm{s}\right), P_{w}$ is the inlet water pressure $(\mathrm{Pa}), \rho_{w}$ is the water density $\left(\mathrm{kg} / \mathrm{m}^{3}\right)$, and $r_{n}$ is the radius of the discharge nozzle $(\mathrm{m})$. In the atomizer test, under an inlet water pressure of $P_{w}=5 \mathrm{bar}$, a flow rate of $3.5 \times 10^{-3} \mathrm{~kg} / \mathrm{s}$ was obtained; the flow rate coefficient was $C_{d}$ $=0.22$. This value of the coefficient was compared to the value that was estimated empirically by Equation (4) [27]:

$$
C_{d}=0.35 \cdot\left(\frac{F_{t}}{D_{s} \cdot d_{n}}\right)^{0.5} \cdot\left(\frac{F_{t}}{d_{n}}\right)^{0.25}
$$

where $F_{t}$ is the inlet tangential cross sectional area $\left(\mathrm{m}^{2}\right), d_{n}$ is the exit nozzle of the swirl chamber $(\mathrm{m})$ and $D_{s}$ is the diameter of the swirl chamber (m). The received flow coefficient $C_{d}$ from Equation (4) is equal to 0.244 . The experimental value of $C_{d}$ is lower than the empirical value, which attests to the optimal choice of the atomizer's geometrical parameters (with minimal hydrodynamic losses and the high possible ratio between the tangential and axial velocities).

Atomization can be represented by the widely used Weber number (We), which estimates when the liquid jet is likely to break up (Equation (5)).

$$
W e=\frac{\rho_{a U_{r} d_{n}}}{\sigma}
$$


where $U_{r}$ is the relative velocity between the liquid jet and ambient $(\mathrm{m} / \mathrm{s}), \rho_{a}$ is the air density $\left(\mathrm{kg} / \mathrm{m}^{3}\right)$, $d_{n}$ is the exit nozzle of the swirl chamber $(\mathrm{m})$, and $\sigma$ is the surface tension $\left(\mathrm{kg} / \mathrm{s}^{2}\right)$. Taking into account the value $U_{r}$ equal to $31.6 \mathrm{~m} / \mathrm{s}$ at $P_{w}=5$ bar, $W e=14$ is considered as favorable condition for the liquid jet break-up (We must be $\gg 1$. The inlet ports Reynolds number is defined as:

$$
R e=\frac{U_{i n} \cdot 2 r_{i n}}{v}
$$

where $U_{i n}$ is the water velocity in the tangential ports $(\mathrm{m} / \mathrm{s}), r_{i n}$ is the radius of the tangential ports $(\mathrm{m})$, and $v$ is the water viscosity $(\mathrm{kg} / \mathrm{m} \cdot \mathrm{s})$.

$$
U=\frac{Q_{w}}{4 \pi \cdot r_{i n}^{2}}
$$

Changing pressure in the diapason of $2-7$ bar, the Reynolds number is in the range of $1.4 \times 10^{4}-2.6$ $\times 10^{4}$. For the given Reynolds numbers, the length of the input ports $\left(l_{t} / d_{t}=2\right)$ is sufficient to completely fill it [38].

The distribution of drop diameters of the mechanical atomizer are presented in Figure 5. At a pressure of 2 bar, the $D_{32}$ of the water drops on the torch axis was $80 \mu \mathrm{m}$. As the distance from the axis of the torch to its periphery (the radius) was increased, the drop diameter rose to $110 \mu \mathrm{m}$. Under increased water pressure the drop diameter in the paraxial zone of the torch decreased, as has been noted by many researchers, and was $58 \mu \mathrm{m}$ at $P_{w}=3$ bar, $48 \mu \mathrm{m}$ at $P_{w}=4$ bar, $40 \mu \mathrm{m}$ at $P_{w}=5$ bar and 37-32 $\mu \mathrm{m}$ at $P_{w}=6-7$ bar. But in the peripheral zone the decrease in the atomized drop diameter as the pressure increases was less significant.

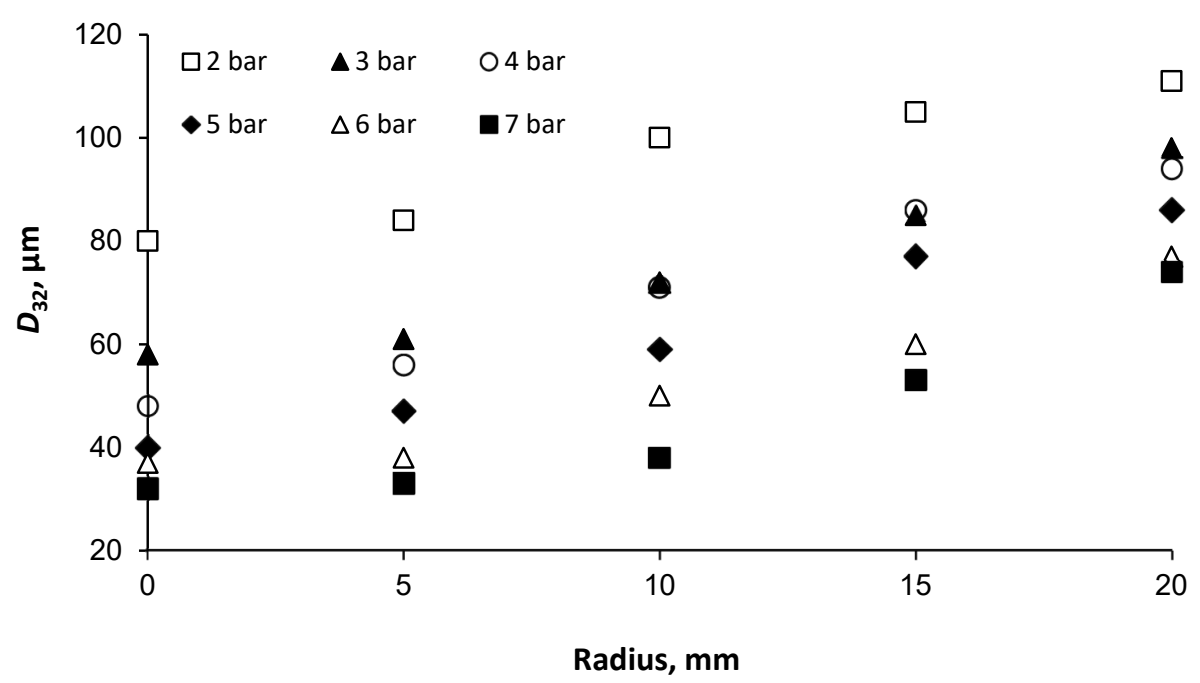

Figure 5. Distribution of $D_{32}$ drop diameters over the radius of the spray torch.

Experiments that were carried out with a Danfoss company atomizer at a water pressure of $P_{w}=8$ bar yielded spray particle diameters of $D_{32}$ equal to $60 \mu \mathrm{m}$ with radius $R=0$, and $115 \mu \mathrm{m}$ with $R=10 \mathrm{~mm}$, while at $P_{w}=13$ bar the spray particle diameters were 40 and $50 \mu \mathrm{m}$, respectively. Rashad et al. investigated the influence of certain geometric ratios of swirl chamber parameters on drop diameters. The authors obtained $D_{32}$ equal to $60-70 \mu \mathrm{m}$ at the supply pressure of $P_{w}=8$ bar, and 50-60 $\mu \mathrm{m}$ at $P_{w}=12$ bar [30]. It must be noted that, according to Xue et al., 58\% of the pressure drop converts into the kinetic energy of the rotational motion in the swirl chamber, an energy loss which includes both hydraulic loss and friction loss [40]. Consequently, optimization of the atomizer swirl chamber geometry can significantly improve the quality of the spray. In addition, the $D_{32}$ drops of the developed mechanical atomizer were calculated using empirical Equation (8) [27] and Equation (9) [47]:

$$
D_{32}=2.25 \sigma^{0.25} \mu_{l}^{0.25} m_{l}^{0.25} \rho_{g}^{-0.25} \Delta P_{l}^{-0.5}
$$




$$
D_{32}=4.4 \sigma^{0.6} \mu_{l}^{0.16} \rho_{l}^{-0.16} m_{l}^{0.22} \Delta P_{l}^{-0.43}
$$

For example, at $P_{w}=5$ bar the $D_{32}$ according to Equations (8) and (9) were expected to be 64 and $82 \mu \mathrm{m}$, respectively. The difference between the empirical and measured results is due to several reasons. First is the limited region in which the PDPA measurements were taken. Droplets can experience secondary breakup and produce smaller droplets further downstream, beyond the range of the physical apparatus at our disposal. Additionally, since the physical phenomena involved in atomization processes are not fully understood, the empirical correlations cannot fully represent the physical principles determining the droplet formation process. Finally, these empirical models were developed based on fuel spray measurements in high pressure conditions such as engine combustion, whereas in our study the sprays operate at a much lower pressure, thus some deviation is inevitable. It must be noted that the $D_{32}$ drops in the pressure range of $2-5$ bar on the torch axis decrease by $50 \%$, but in the range of $6-7$ bar they decrease only by $13.5 \%$. Therefore, when using an atomizer with the optimal geometrical dimensions as we have stated, the fluid supply pressure should be limited to 5 bar.

The standard histograms for the distribution of drop diameters and velocities in the paraxial zone $(R=0)$ are presented in Figure 6. As can be seen from the data obtained, the peak of the histogram occurs within 15-25 $\mu \mathrm{m}$. This is in accordance with the finding of Chang et al. [48], who reported that the smaller drops are mostly confined to the core regions of the spray. The larger drops, more than $40 \mu \mathrm{m}$, occupy the peripheral part of the spray.

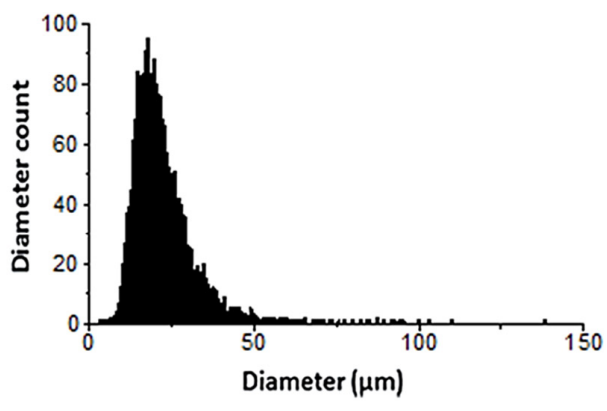

(a)

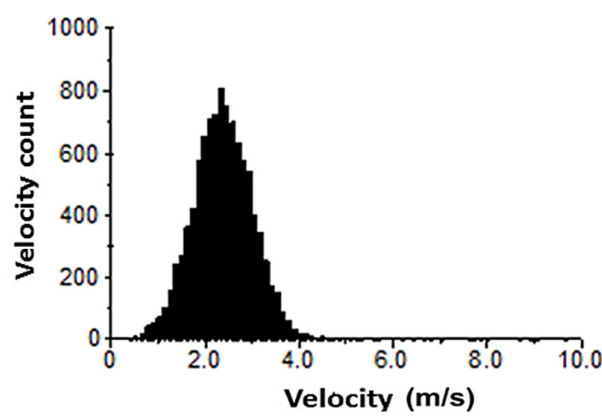

(b)

Figure 6. Diameter (a) and velocity (b) histograms at $P_{w}=4$ bar, $R=0 \mathrm{~mm}$.

The droplets' axial velocity distribution helps to facilitate the expansion of the spray, and the diffusion to the ambient air, which is more intense in the downstream region of the spray where it promotes turbulent mixing. In Figure 7 the change in axial drop velocity is shown for pressures of 2 and 3 bar, where bigger drop diameters are produced, and for pressures 6 and 7 bar, where smaller drop diameters are produced.

As can be seen from these measurements, in the cross section at $50 \mathrm{~mm}$ downstream from the nozzle, most droplets have a velocity ranging from $3 \mathrm{~m} / \mathrm{s}$ to $5.5 \mathrm{~m} / \mathrm{s}$. This figure also illustrates that droplets of different sizes at various locations, can have the same velocities depending on their trajectory. However, most of them are in a speed range that gives them sufficient momentum to penetrate the surrounding medium. A higher water supply pressure, together with smaller droplets (pressure 6-7 bar), yields higher velocities because of their larger drag-to-momentum ratio. The initial discrepancies between the velocities of various sizes drops are preserved over the entire jet torch cross section.

The generated spray of a cone-shaped pattern of volume flux is illustrated in Figure 8, which is characterized by the high concentration of water drops at the edges of the spray cone and low concentration in the central part of the spray.

It is essential to know the spatially distributed flux density of a mechanical atomizer for the design of a spray system. These measurements are complementary to the drop size, the coefficient of discharge and the spray cone angle. From the obtained data shown in Figure 5, the smallest drops are located 
in the central part of the spray cone. These small-sized drops have a large surface/volume ratio, and evaporate more quickly than the larger ones, which is why the central part of the spray has a low drop concentration. The volume flux in the central part is at a minimum and decreases with increased water pressure, which yields the smallest drop sizes. For example, the volume flux in the central part was $0.007 \mathrm{~cm}^{3} /\left(\mathrm{cm}^{2} \cdot \mathrm{s}\right)$ at $P_{w}=5 \mathrm{bar}$, and $0.032 \mathrm{~cm}^{3} /\left(\mathrm{cm}^{2} \cdot \mathrm{s}\right)$ at $P_{w}=2$ bar. Because large drops evaporate with relative difficulty and hence accumulate at the edge of the spray, the bigger flux volume is concentrated on the jet periphery, as is shown in Figure 8. In the peripheral region the jet volume flux is equal to $0.075 \mathrm{~cm}^{3} /\left(\mathrm{cm}^{2} \cdot \mathrm{s}\right)$ at $P_{w}=2 \mathrm{bar}$, and $0.106 \mathrm{~cm}^{3} /\left(\mathrm{cm}^{2} \cdot \mathrm{s}\right)$ at $P_{w}=5$ bar. Additionally, the spray angle slightly increases with increased water pressure, as seen in Figure 9 , and is in the range $\beta=65^{\circ}-78^{\circ}$.

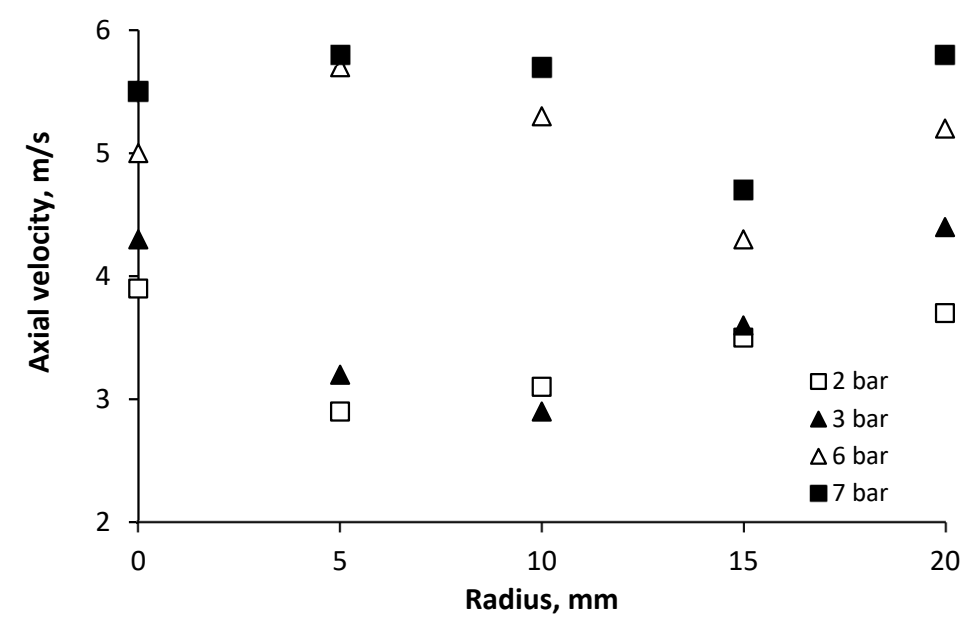

Figure 7. Axial droplet velocity vs radius for different water pressures.

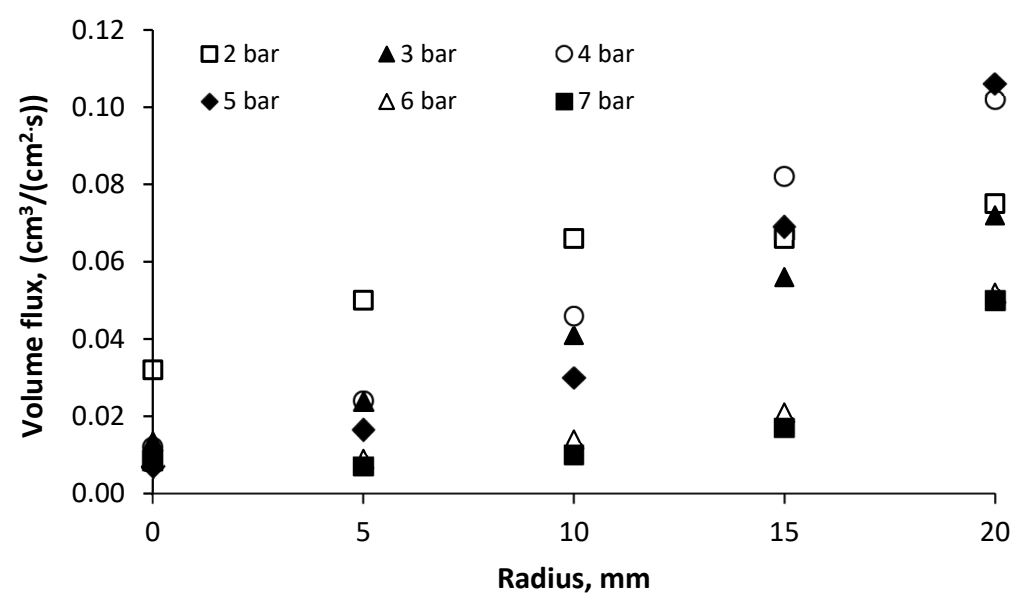

Figure 8. Volume flux vs radius for different water pressure.

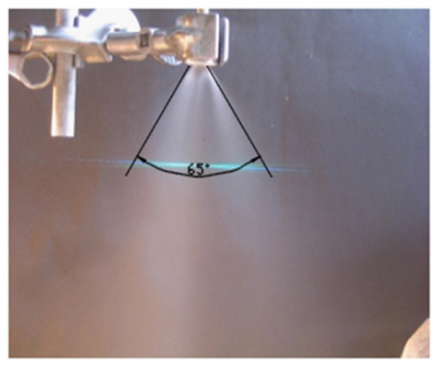

(a)

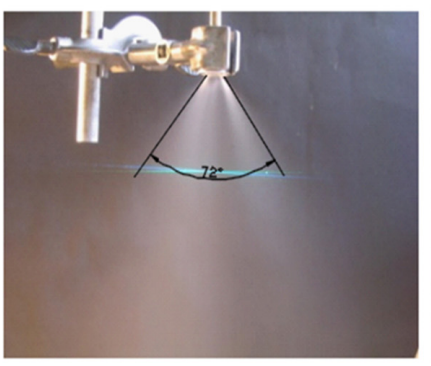

(b)

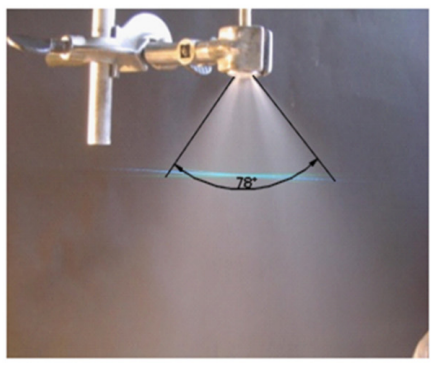

(c)

Figure 9. The spay torch angle $(\beta)$ of the mechanical atomizer at varying water pressures: (a) 3 bar, (b) 4 bar, (c) 5 bar. 


\subsection{The Air-Assisted Atomizer}

To obtain small diameters of liquid droplets at low supply pressures, a gas flow is introduced into the atomizer. In our studies, a liquid atomizer with an optimal geometry was installed at the first stage for liquid spraying. At the second stage the effluent conical jet interacts with the air flow which is introduced through the tangential channels of the swirl chamber. The test results of the mechanical atomizer that provides preliminary water spray in the air-assisted atomizer with different exit nozzle diameters $d_{n}=0.6 \mathrm{~mm}$ and $0.8 \mathrm{~mm}$ are shown in Figure 10 .

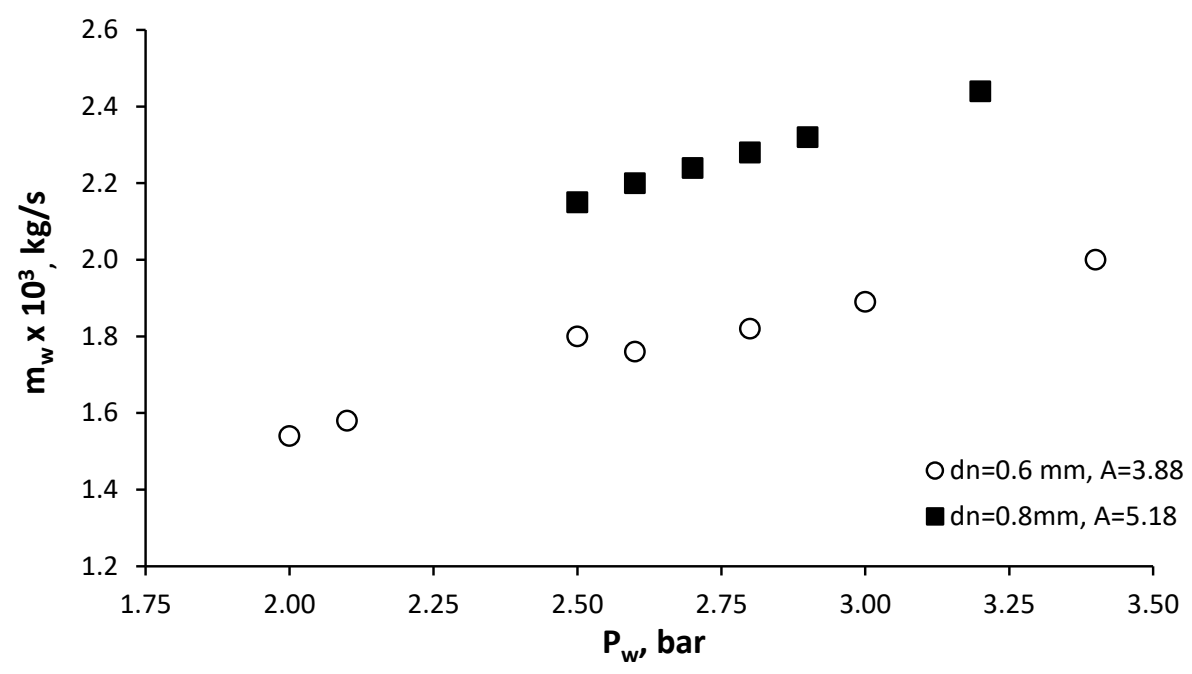

Figure 10. Water flow rate characteristic of the mechanical atomizer.

The flow rate coefficient $C_{d}$ of the mechanical atomizer, calculated by Equation (3), was equal to 0.27 and 0.19 with the nozzle diameters $0.6 \mathrm{~mm}$ and $0.8 \mathrm{~mm}$, respectively. With the air flow, using a channel diameter of $0.6 \mathrm{~mm}$, the value of $A$ was higher than the value was without the air flow (3.88 vs. 3.2). The calculated value of $C_{d}$ in the presence of air flow was actually higher, though we expected it to be lower. This is explained by a significantly larger value $l_{0} / d_{n}$ (3.3 and 2.5), leading to an increase in hydraulic losses, which corresponds to the data obtained in $[32,38]$. The Reynolds number (Equation (6)) for flow from the exit nozzle at operating parameters was in the range $8.5 \times 10^{3}-1.1 \times$ $10^{4}$ for diameter $d_{n}=0.6 \mathrm{~mm}$ and $1.1 \times 10^{4}-1.5 \times 10^{4}$ for $d_{n}=0.8 \mathrm{~mm}$. The values of coefficient $C_{d}$ for these test variants were also determined in accordance to Equation (4) and found $C_{d}=0.23$ for $d_{n}=0.6 \mathrm{~mm}, C_{d}=0.185$ for $d_{n}=0.8 \mathrm{~mm}$, which approximates to the experimental data.

Air flow rate characteristics of the swirl chamber are shown in Figure 11.

The test results show that the air flow rate through the swirl chamber is determined not only by its supply pressure and the diameter of the tangential channels, but also by the operating parameters of the mechanical atomizer. This can be explained by the influence of the torch angle of the liquid jet and its energy, which, when interacting with the air flow at the outlet of the tangential channels, has a certain resistance to its flow. For example, at $P_{a}=1$ bar and in a mechanical atomizer with a diameter of the exit nozzle of $d_{n}=0.6 \mathrm{~mm}$ at $P_{w}=2.5 \mathrm{bar}$, the air flow rate was $2.2 \times 10^{-3} \mathrm{~kg} / \mathrm{s}\left(d_{t}=1.8 \mathrm{~mm}\right)$. Using these operating conditions for the air swirl chamber but with mechanical atomizer parameters set at $d_{n}=0.8 \mathrm{~mm}$ and $P_{w}=3.2 \mathrm{bar}$, the air flow rate was $1.95 \times 10^{-3} \mathrm{~kg} / \mathrm{s}(-11.5 \%)$. At $d_{t}=2 \mathrm{~mm}$, $P_{a}=0.7$ bar and for a mechanical atomizer with $d_{n}=0.6 \mathrm{~mm}$, water supply pressure $P_{w}=2.6$ bar, the air flow rate was $1.88 \times 10^{-3} \mathrm{~kg} / \mathrm{s}$, but when the nozzle was set to $d_{n}=0.8 \mathrm{~mm}$ and $P_{w}=2.7$ bar the air flow rate was $1.73 \times 10^{-3} \mathrm{~kg} / \mathrm{s}(-8 \%)$. As can be seen from Figure 11, increasing the diameter of the nozzle $d_{n}$ reduces the air flow rate. This corresponds to the influence of the liquid jet torch angle which 
is determined by the geometrical characteristic $A$. According to theory [39], the torch angle $\beta$ increases with increasing the characteristic $A$ :

$$
\operatorname{tg} \beta / 2=\frac{2 C_{d} \cdot A}{\sqrt{\left(1+\frac{r_{\varphi}}{d_{n} / 2}\right)^{2}-4 C_{d}^{2} A^{2}}}
$$

where $r_{\varphi}$ is a cavity zone radius (mm) and equal $r_{\varphi}=d_{n} / 2 \sqrt{1-\varphi_{n}}$.

The $\varphi_{n}$ is determined by:

$$
A=\frac{\left(1-\varphi_{n}\right) \sqrt{2}}{\varphi_{n} \sqrt{\varphi_{n}}}
$$

This is in accordance with the calculation for amechanical atomizer with $d_{n}=0.6 \mathrm{~mm} \beta / 2=48^{\circ}$, with $d_{n}=0.8 \mathrm{~mm} \beta / 2=62^{\circ}$. Thus, an increase in the jet torch angle at the exit of the mechanical atomizer reduces the air flow rate.

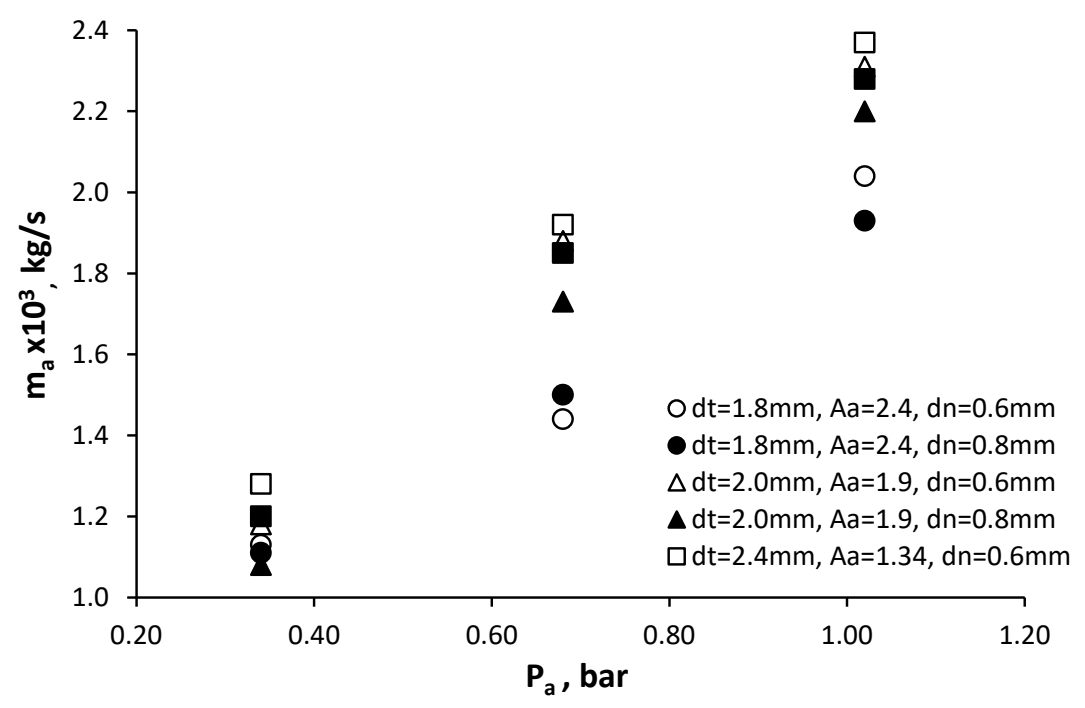

Figure 11. Air flow rate characteristics of the swirl chamber.

Introducing swirling air flow at the exit of the mechanical atomizer affects the radial velocity component. This effect should lead to a widening of the spray cone angle and have a significant influence on the drop size. The spay torch angle of the air-assisted atomizer increases to $\beta=90^{\circ}-95^{\circ}$ and is shown in Figure 12.

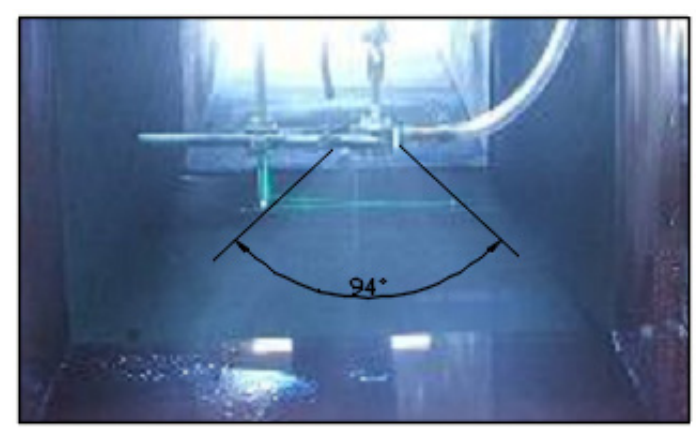

Figure 12. The spray torch angle of an air-assisted atomizer.

One of the parameters determining spray quality in the air-assisted atomizer is the air to water flow ratio (ALR). The distribution drop sizes of some versions of the atomizer assembly are given in Figure 13. 


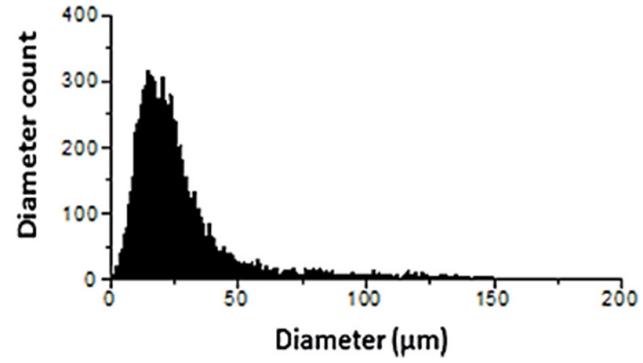

(a)

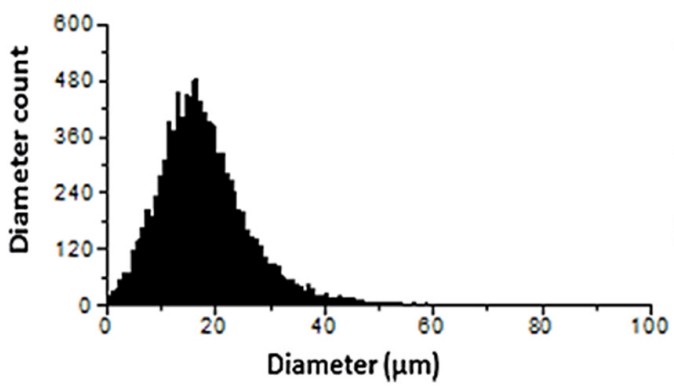

(c)

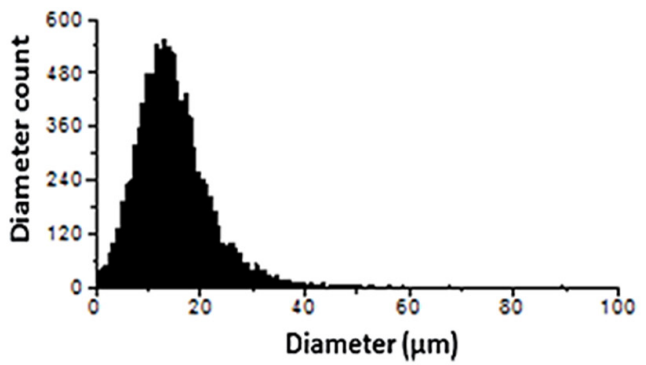

(b)

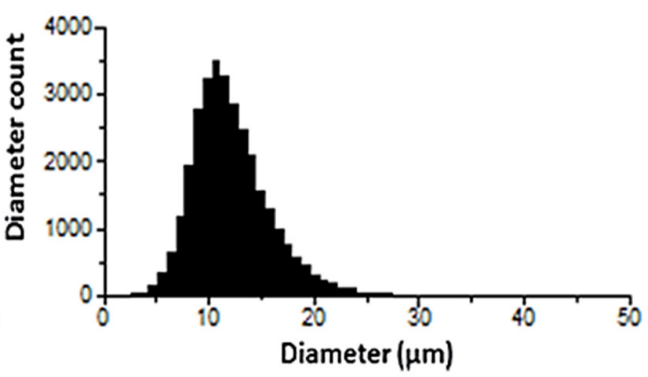

(d)

Figure 13. The influence of operation conditions on the atomization quality of the atomizer assembly. (a) $d_{t}=2.0 \mathrm{~mm}, d_{n}=0.6 \mathrm{~mm}, A L R=0.69, P_{w}=2.5$ bar, $P_{a}=0.35$ bar; (b) $d_{t}=2.0 \mathrm{~mm}, d_{n}=0.6 \mathrm{~mm}$, $A L R=1.19, P_{w}=3$ bar, $P_{a}=1$ bar; (c) $d_{t}=2.4 \mathrm{~mm}, d_{n}=0.8 \mathrm{~mm}, A L R=0.67, P_{w}=2.5$ bar, $P_{a}=0.35$ bar; (d) $d_{t}=2.4 \mathrm{~mm}, d_{n}=0.8 \mathrm{~mm}, A L R=0.97, P_{w}=2.9$ bar, $P_{a}=1$ bar.

As can be seen from Figure 13, the introduction of a swirling air flow at the exit of the jet from the mechanical atomizer significantly reduces the diameter of the droplets, even at low supply pressures for water and air. Moreover, with an increase in ALR, the diameter of the droplets decreases to $20-30 \mu \mathrm{m}$.

The dependence of $D_{32}$ water drop diameters on the ALR of an operating air swirl chamber that has various tangential channels and that uses a mechanical atomizer equipped with various exit nozzles is shown in Figure 14.

Installing the air swirl chamber substantially decreases drop sizes, as these are affected by the energy of the air. For all ranges of values of ALR $=0.47-1.25$ and $P_{w}=2-3.4$ bar, the $D_{32}$ drops were in the range of 25-48 $\mu \mathrm{m}$ respectively (using only the mechanical atomizer, the $D_{32}$ ranged from 58 to $110 \mu \mathrm{m}$ at $P_{w}=2-3$ bar). The test results show that the geometrical characteristic of the air swirl chamber $\left(A_{\mathrm{a}}=1.34-2.4\right)$ does not exert a considerable influence upon atomization quality. At the same time, the geometrical characteristic $A$ of the mechanical atomizer does influence the results via the quality of the preliminary atomization. Thus, a mechanical atomizer with $A=5.18\left(d_{n}=0.8 \mathrm{~mm}\right)$ has all the necessary prerequisites for producing more finely dispersed water atomization. For example, the $D_{32}$ drop diameters equal to $35 \mu \mathrm{m}$ at ALR $=1.13$ were obtained in the test with the air swirl chamber when the tangential ducts were set at $d_{t}=1.8 \mathrm{~mm}$ and the nozzle diameter at $0.6 \mathrm{~mm}$; but with the nozzle diameter set at $d_{n}=0.8 \mathrm{~mm}$, results were $D_{32}=30 \mu \mathrm{m}$ at ALR $=0.8$ (Figure 14). In the test with the air swirl chamber with tangential channels diameter $d_{t}=2 \mathrm{~mm}$ and nozzle $d_{n}=0.6 \mathrm{~mm}$, the $D_{32}$ drop diameter was equal to $48 \mu \mathrm{m}$ at $\mathrm{ALR}=0.69$, while with the nozzle set at $d_{n}=0.8 \mathrm{~mm}, D_{32}=45 \mu \mathrm{m}$ was obtained at ALR $=0.54$. It must be noted that in the test of the air-assisted atomizer in [49], $D_{32}$ of $40-35 \mu \mathrm{m}$ was obtained at ALR $=5-9$.

Finally, it should be noted that an air supply pressure of over 1 bar does not have a significant influence upon the quality of atomization. This two-phase atomizer provides small diameters of spray droplets at significantly lower air supply pressures than two-phase atomizers of well-known companies. Since the compressed air supply pressure has the main effect on the energy consumption 
compared to liquid pressure, the use of this atomizer will reduce the energy cost. Consequently, we are able to recommend an air and water flow rates ratio setting of ALR $\approx 1$.

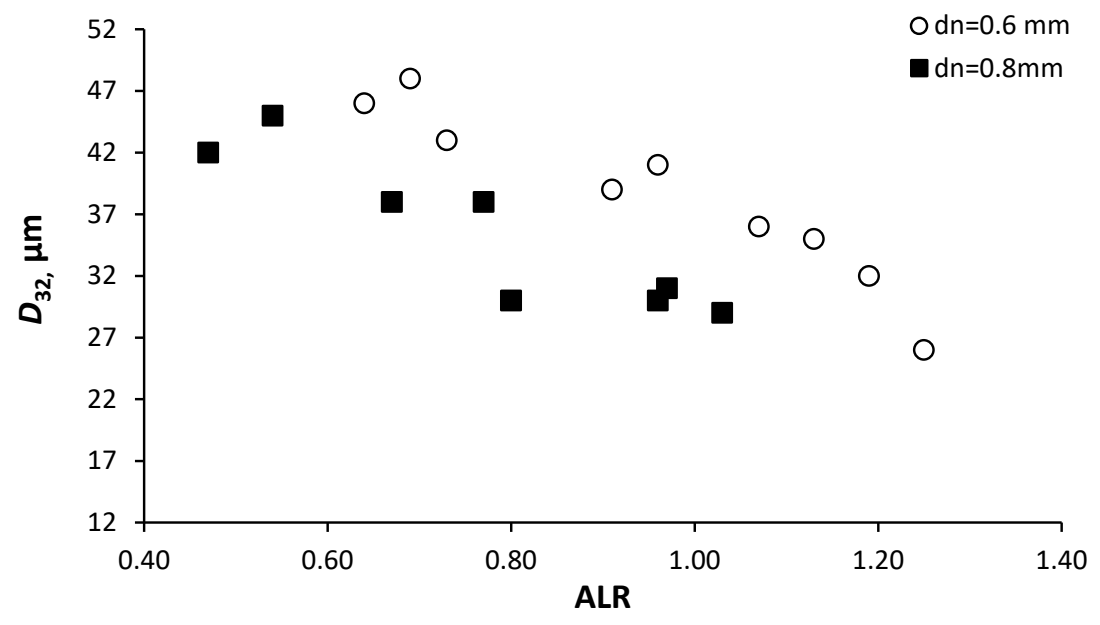

Figure 14. Dependence of $D_{32}$ drop diameters on ALR.

\section{Conclusion}

The possibility of improving liquid atomization by using a mechanical atomizer and introducing an air-assistance component has been investigated. We have shown that with optimization of the atomizer's geometric parameters, it is possible to obtain drop diameters of $D_{32}=80 \mu \mathrm{m}$ at a water supply pressure of $P_{w}=2$ bar, and $D_{32} \approx 40 \mu \mathrm{m}$ at $P_{w}=4-5$ bar. In this design variant, further increases in pressure beyond 5 bar do not, practically speaking, influence the atomization quality.

This high-quality atomization was obtained with a low consumption of energy by using an air-assisted component, with the mechanical atomizer optimized for its geometric parameters installed as the first stage for preliminary spray of the water, and with a swirl air flow interaction supplied at low pressure as the second stage. Results obtained from the experimental test produced drop diameters of $D_{32}=20-30 \mu \mathrm{m}$ at the water pressures of 2.5-3 bar and air-supply pressures of $0.35-1$ bar, and at $A L R \approx 1$. The drop diameters decrease if the ALR is increased. Further increasing air-supply pressure beyond 1 bar does not result in significant decreases in water drop diameters. The supply of an air flow increases the spray torch angle to $90^{\circ}-95^{\circ}$.

From this study's results, it can be seen that the micro-droplet atomizer works in wide range of flow rate and low inlet pressure of liquid and gas. This suits for development of liquid fuels micro-turbine and other generator systems.

Author Contributions: Conceptualization, I.L. and D.T.; methodology, I.L. and D.T; formal analysis, I.L. and D.T.; investigation, I.L. and D.T; resources, I.L. and D.T.; data curation, I.L. and D.T.; writing-original draft preparation, I.L. and D.T; writing - review and editing, I.L. and D.T; visualization, I.L. and D.T.; supervision, I.L. and D.T; project administration, I.L. and D.T; funding acquisition, I.L. and D.T. All authors have read and agreed to the published version of the manuscript.

Funding: This research received no external funding.

Conflicts of Interest: The authors declare no conflict of interest.

\section{References}

1. Yao, C.; Geng, P.; Yin, Z.; Hu, J.; Chen, D.; Ju, Y. Impacts of nozzle geometry on spray combustion of high pressure common rail injectors in a constant volume combustion chamber. Fuel 2016, 179, 235-245. [CrossRef]

2. Williams, A. Combustion of Liquid Fuel Sprays; Butterworth-Heinemann: London, UK, 1990.

3. Li, S.; Willits, D.H. Comparing low-pressure and high-pressure fogging systems in naturally ventilated greenhouses. Biosyst. Eng. 2008, 101, 69-77. [CrossRef] 
4. Xu, Z.; Xiao, Y.; Wang, Y. Experimental and theoretical studies on air humidification by a water spray at elevated pressure. Appl. Therm. Eng. 2007, 27, 2549-2558. [CrossRef]

5. Nederhoff, E.; van Weel, P. Humidification: Fogging and Other Evaporative Cooling in Greenhouses. Pract. Hydroponics Greenh. 2011, 118, 46-52.

6. Guo, Q.; Ren, W. Atomization parameters and fire suppression characteristics of water mist in simulated roadway. Geomat. Nat. Haz. Risk. 2019, 10, 1529-1541. [CrossRef]

7. Kunyoung, L.E.E.; Kim, T.; Jongsu, L.E.E.; Choi, J.; Sanghyuk, S.O.N.; Lee, K. Apparatus for Both Humidification and Air Cleaning. U.S. Patent No. 10094582, 9 October 2018.

8. LeFort, G.; Marshall, A.W.; Pabon, M. Evaluation of surfactant enhanced water mist performance. Fire Technol. 2009, 45, 341-354. [CrossRef]

9. Sharma, A.; Jana, A.H.; Chavan, R.S. Functionality of milk powders and milk-based powders for end use applications-A review. Compr. Rev. Food Sci. Food Saf. 2012, 11, 518-528. [CrossRef]

10. Vervaet, C.; Remon, J.P. Continuous granulation in the pharmaceutical industry. Chem. Eng. Sci. 2005, 60, 3949-3957. [CrossRef]

11. Ochman, J.; Bialik, W.; Gil, S. An experimental study on liquid fuel atomization. Metalurgija 2015, 54, 559-562.

12. Sponza, D.T. Toxicity studies in a tobacco industry biological treatment plant. Water Air Soil Pollut. 2002, 134, 137-164. [CrossRef]

13. Marchione, T.; Allouis, C.; Amoresano, A.; Beretta, F. Experimental investigation of a pressure swirl atomizer spray. J. Propul. Power. 2007, 23, 1096-1101. [CrossRef]

14. Bian, J.; Zhang, D.; Sun, R.; Wu, Y.; Tian, W.; Su, G.H.; Qiu, S. Experimental study on spray characteristics of pressure-swirl nozzle in China advanced PWR containment. Nucl. Eng. Des. 2019, 350, 158-175. [CrossRef]

15. Chaker, M.A.; Meher-Homji, C.B.; Mee III, T. Inlet fogging of gas turbine engines: Experimental and analytical investigations on impaction pin fog nozzle behavior. J. Eng. Gas Turbines Power. 2006, 128, 826-839. [CrossRef]

16. Chaker, M.; Meher-Homji, C.B.; Mee, T., III; Nicholson, A. Inlet fogging of gas turbine engines detailed climatic analysis of gas turbine evaporation cooling potential in the USA. J. Eng. Gas Turbines Power 2003, 125, 300-309. [CrossRef]

17. Lefebvre, A.H. The role of fuel preparation in low-emission combustion. J. Eng. Gas Turbines Power. 1995, 117, 617-654. [CrossRef]

18. Roudini, M.; Wozniak, G. Experimental investigation of spray characteristics of pre-filming air-blast atomizers. J. Appl. Fluid Mech. 2018, 11, 1455-1469. [CrossRef]

19. Jedelsky, J.; Jicha, M.; Slama, J.; Otahal, J. Development of an effervescent atomizer for industrial burners. Energy Fuel 2009, 23, 6121-6130. [CrossRef]

20. Lefebvre, A.H. Fifty years of gas turbine fuel injection. At. Spray 2000, 10, 251-276. [CrossRef]

21. Bayrel, L. Orzechowski, Z. Liquid Atomization; Taylor \& Francis: Washington, DC, USA, 1993.

22. Shraiber, A.A.; Podvysotsky, A.M.; Dubrovsky, V.V. Deformation and breakup of drops by aerodynamic forces. At. Spray 1996, 6, 667-692. [CrossRef]

23. Han, Z.; Parrish, S.E.; Farrell, P.V.; Reitz, R.D. Modeling atomization processes of pressure-swirl hollow-cone fuel sprays. At. Spray 1997, 7, 663-684. [CrossRef]

24. Lefebvre, A.H.; McDonell, V.G. Atomization and Sprays; CRC Press: Boca Raton, FL, USA; Taylor \& Francis: Washington, DC, USA, 2017.

25. Dafsari, R.A.; Lee, H.J.; Han, J.; Park, D.C.; Lee, J. Viscosity effect on the pressure swirl atomization of an alternative aviation fuel. Fuel 2019, 240, 179-191. [CrossRef]

26. Santangelo, P.E. Characterization of high-pressure water-mist sprays: Experimental analysis of droplet size and dispersion. Exp. Therm. Fluid Sci. 2010, 34, 1353-1366. [CrossRef]

27. Rizk, N.K.; Lefebvre, A.H. Internal flow characteristics of simplex swirl atomizers. J. Propul. Power 1985, 1, 193-199. [CrossRef]

28. Lee, J.D.; Saha, A.; Basu, S.; Kumar, R. Effects of Injection Pressure on Spray Atomization Characteristics with Measurement Technique Cross-validation. In Proceedings of the 12th Triennial International Conference on Liquid Atomization and Spray Systems, Heidelberg, Germany, 2-6 September 2012.

29. Włodarczak, S.; Ochowiak, M.; Matuszak, M. Atomizers with the swirl motion phenomenon. Pract. Aspects Chem. Eng. 2018, 437-452. 
30. Rashad, M.; Yong, H.; Zekun, Z. Effect of geometric parameters on spray characteristics of pressure swirl atomizers. Int. J. Hydrogen Energy 2016, 41, 15790-15799. [CrossRef]

31. Dar, U.A.; Bannikov, M. Swirl atomizer design for evaporative cooling of high temperature compressed air stream. Int. J. Fluid Mech. Res. 2014, 41, 51-70. [CrossRef]

32. Zhang, T.; Dong, B.; Chen, X.; Qiu, Z.; Jiang, R.; Li, W. Spray characteristics of pressure-swirl nozzles at different nozzle diameters. Appl. Therm. Eng. 2017, 121, 984-991. [CrossRef]

33. Elkotb, M.M.; Rafat, N.M.; Hanna, M.A. The Influence of Swirl Atomizer Geometry on the Atomization Performance. In Proceedings of the 1st International Conference on Liquid Atomization and Spray Systems, Hanna, Tokyo, Japan, 27-31 August 1978.

34. Sakman, A.T.; Jog, M.A.; Jeng, S.M.; Benjamin, M.A. Parametric study of simplex fuel nozzle internal flow and performance. AIAA J. 2000, 38, 1214-1218. [CrossRef]

35. Chen, S.K.; Lefebvre, A.H.; Rollbuhler, J. Factors influencing the effective spray cone angle of pressure-swirl atomizers. J. Eng. Gas Turbines Power 1992, 114, 97-103. [CrossRef]

36. Ochowiak, M.; Lytvynenko, O.; Włodarczak, S.; Matuszak, M.; Krupińska, A. Design and study of conical pressure-swirl atomizers. DSMIE 2019, 472-480.

37. Lee, S.G. Geometrical effects on spray characteristics of air-pressurized swirl flows. J. Mech. Sci. Technol. 2008, 22, 1633-1639. [CrossRef]

38. Yule, A.J.; Widger, I.R. Swirl atomizers operating at high water pressure. Int. J. Mech. Sci. 1996, 38, 981-999. [CrossRef]

39. Khavkin, Y.I. Theory and Practice of Swirl Atomizers; CRC Press: Boca Raton, FL, USA, 2003.

40. Xue, J.; Jog, M.A.; Jeng, S.M.; Steinthorsson, E.; Benjamin, M.A. Effect of geometric parameters on simplex atomizer performance. AIAA J. 2004, 42, 2408-2415. [CrossRef]

41. Rizkalla, A.A.; Lefebvre, A.H. The influence of air and liquid properties on airblast atomization. J. Fluids Eng. 1975, 97, 316-320. [CrossRef]

42. Fraser, R.P.; Dombrowski, N.; Routley, J.H. The atomization of a liquid sheet by an impinging air stream. Chem. Eng. Sci. 1963, 18, 339-353. [CrossRef]

43. Levy, Y.; Sherbaum, V.; Levin, D.; Ovcharenko, V. Airblast Swirl Atomizer for Small Jet Engines. In Proceedings of the ASME Turbo Expo 2005: Power for Land Sea, and Air, Reno, NV, USA, 6-9 June 2005.

44. Albrecht, H.E.; Borys, M.; Damaschke, N.; Tropea, C. Laser Doppler and Phase Doppler MEASUREMENT Techniques; Springer: Berlin, Germany, 2003.

45. Black, D.L.; McQuay, M.Q.; Bonin, M.P. Laser-based techniques for particle-size measurement: A review of sizing methods and their industrial applications. Progr. Energy Combust. Sci. 1996, 22, 267-306. [CrossRef]

46. Valencia-Bejarano, M.; Langrish, T.A.G. Experimental investigation of droplet coalescence in a full-cone spray from a two-fluid nozzle using laser diffraction measurements. At. Spray 2004, 14, 355-374. [CrossRef]

47. Wei, X.; Yong, H. Improved semiempirical correlation to predict sauter mean diameter for pressure-swirl atomizers. J. Propul. Power 2014, 30, 1628-1635. [CrossRef]

48. Chang, K.C.; Wang, M.R.; Wu, W.J.; Hong, C.H. Experimental and theoretical study on hollow-cone spray. J. Propul. Power 1993, 9, 28-34. [CrossRef]

49. Kourmatzis, A.; Pham, P.X.; Masri, A.R. Air assisted atomization and spray density characterization of ethanol and a range of biodiesels. Fuel 2013, 108, 758-770. [CrossRef]

(C) 2020 by the authors. Licensee MDPI, Basel, Switzerland. This article is an open access article distributed under the terms and conditions of the Creative Commons Attribution (CC BY) license (http://creativecommons.org/licenses/by/4.0/). 